\title{
Three-Dimensional Multiscale Modeling of Dendritic Spacing Selection During Al-Si Directional Solidification
}

\author{
DAMIEN TOURRET $\odot,{ }^{1,3}$ AMY J. CLARKE, ${ }^{1}$ SETH D. IMHOFF ${ }^{1}$ \\ PAUL J. GIBBS, ${ }^{1}$ JOHN W. GIBBS,${ }^{1}$ and ALAIN KARMA ${ }^{2}$
}

1.-Materials Science and Technology Division, Los Alamos National Laboratory, Los Alamos, NM 87545, USA. 2.-Department of Physics and Center for Interdisciplinary Research on Complex Systems, Northeastern University, Boston, MA 02115, USA. 3.—e-mail: dtourret@lanl.gov

We present a three-dimensional extension of the multiscale dendritic needle network (DNN) model. This approach enables quantitative simulations of the unsteady dynamics of complex hierarchical networks in spatially extended dendritic arrays. We apply the model to directional solidification of $\mathrm{Al}$ 9.8 wt.\% Si alloy and directly compare the model predictions with measurements from experiments with in situ x-ray imaging. We focus on the dynamical selection of primary spacings over a range of growth velocities, and the influence of sample geometry on the selection of spacings. Simulation results show good agreement with experiments. The computationally efficient DNN model opens new avenues for investigating the dynamics of large dendritic arrays at scales relevant to solidification experiments and processes.

\section{INTRODUCTION}

Dendritic microstructures are ubiquitous to cast metals and alloys. The geometrical features of these structures are crucial to mechanical properties of a cast part, and subsequently to its performance during service. ${ }^{1}$ For instance, the primary spacing between main dendrite trunks has a fundamental influence on the strength of a dendritic grain. Hence, being able to understand the selection mechanisms and to predict the spacings resulting from given solidification conditions are key to explore and develop innovative alloys and processes.

Early experiments suggested that the primary spacing $\Lambda$ depends on the growth (or pulling) velocity $V_{\mathrm{p}}$ as $\Lambda \sim V_{\mathrm{p}}^{-v}$ with an exponent $v=0.5^{2}$ or $0.25{ }^{3,4}$ It was later shown that, even though a similar history leads to a similar spacing, ${ }^{5-7}$ a wide range of spacings may be selected for the same control parameters. ${ }^{8,9}$ In particular, primary spacing evolution exhibits a hysteretic dependence with a change of growth velocity. ${ }^{10} \mathrm{~A}$ semi-analytical model has been proposed by Warren and Langer to predict the primary spacing that is dynamically selected through a sequence of primary branch elimination processes, which causes the array spacing to increase transiently in time after morphological instability of the planar interface until reaching a steady-state value. ${ }^{11}$ Other models of spacing selection have been proposed based on geometrical arguments. ${ }^{12,13}$ Those latter models assume steady-state growth dynamics and estimate the upper limit of stable spacing $\Lambda_{\max }$ to be about twice the lower limit $\Lambda_{\min },{ }^{12}$ while recent numerical studies have predicted a ratio $\Lambda_{\max } / \Lambda_{\min }$ closer to three or four. ${ }^{14,15}$ (For a brief review of primary spacing selection theory, see Sect. 2.1 of Ref. 16).

On a larger scale, models based on volume-averaged methods such as cellular automata coupled with finite elements ${ }^{17,18}$ have been developed to predict the grain structure of castings. Those models describe the stochastic nucleation of new grains and are therefore able to model complex phenomena such as the columnar-to-equiaxed transition. ${ }^{19}$ However, they only predict grain envelope shapes and rely on phenomenological scaling laws for dendritic spacings to estimate key parameters such as the solutal diffusion length within a grain.

On a dendrite scale, phase-field (PF) modeling has established itself over the past two decades as the method of choice to predict complex solid-liquid interface patterns. ${ }^{20}$ Theoretical developments of the thin interface limit ${ }^{21}$ and the antitrapping current $^{22}$ opened the way to quantitative modeling of alloy solidification. Additionally, the fast increase in computational resources and the development of 
advanced numerical methods, such as adaptative remeshing ${ }^{23}$ and massive parallelization, ${ }^{24,25}$ have allowed PF simulations up to the scale of threedimensional arrays of cells ${ }^{26}$ or dendrites. ${ }^{25}$

Phase-field simulations are thus capable of predicting stable dendritic spacing ranges in the steady state, ${ }^{14,26-28}$ as well as the dynamical selection of spacings by elimination during the transient stage of solidification. ${ }^{28-30}$ Recent $\mathrm{PF}$ studies have also shown that, in polycrystalline columnar growth, the dynamical evolution of dendritic spacing at converging grain boundaries is closely linked to the unusual overgrowth mechanism of a favorably oriented grain. ${ }^{16,31,32}$

While PF remains the method of choice for simulation of dilute alloy solidification, quantitative predictions of dendritic growth dynamics with $\mathrm{PF}$ require an accurate morphological description of each dendrite tip. This makes PF simulations extremely challenging for concentrated alloys that usually solidify as a hierarchical network of welldeveloped branches (Fig. 1a) with several orders of magnitude separating the scale of a dendrite tip radius and the larger scale of diffusive transport in the melt.

For such multiscale problems, we developed in two dimensions (2D) a dendritic needle network (DNN) approach ${ }^{15}$ that is aimed at bridging the length scale gap between phase-field and volumeaveraged models. We recently extended this model to three dimensions (3D). ${ }^{34}$ DNN simulations reproduce well-know steady-state solutions, as well as transient early stages of equiaxed growth in both $2 \mathrm{D}^{15}$ and $3 \mathrm{D} .^{34} \mathrm{In} 2 \mathrm{D}$, where direct comparison with $\mathrm{PF}$ is still achievable, the model provides reasonable quantitative agreement with $\mathrm{PF}$ predictions for transient isothermal growth dynamics. Furthermore, DNN predictions remain quantitative up to a numerical grid spacing comparable to the dendrite tip radius, which is typically one order of magnitude larger than the grid spacing required to resolve the spatially diffuse interface in PF models. Hence, when both DNN and PF models are discretized on a finite-difference grid and the diffusion equation is solved by explicit time stepping, DNN simulations are faster than PF simulations by four to five orders of magnitude in $2 \mathrm{D}$ and $3 \mathrm{D}$, respectively.

In this paper, we illustrate the application of the 3D DNN model with the example of directional solidification of Al-9.8 wt.\% Si alloy and we compare modeling predictions with experiments for different growth velocities. We summarize in the next section the DNN model for directional solidification in 3D. We then describe the experimental procedure for in situ x-ray imaging during $\mathrm{Al}-\mathrm{Si}$ directional solidification, and present specific simulations performed for quantitative comparison with experiments. Finally, we discuss primary spacing selection as a function of growth velocity, as well as the influence of mold geometry for given thermal conditions.

\section{DENDRITIC NEEDLE NETWORK MODEL}

In concentrated alloys, dendritic grains often grow as hierarchical structures of sharp needle-like branches, as illustrated in Fig. 1a. In the DNN model, such a grain is represented by a network of thin branches, as in Fig. 1b. In the diffusive regime, the solute concentration in the melt $c$ follows

$$
\partial_{t} c=D \nabla^{2} c,
$$

with $D$ the diffusion coefficient. Assuming diffusion is negligible in the solid phase, the solid-liquid interface locally grows at a velocity $v_{\text {n }}$ given by

$$
(1-k) c_{1} v_{\mathrm{n}}=-\left.D \partial_{\mathrm{n}} c\right|_{\mathrm{i}}
$$

with $k$ the interface solute partition coefficient, $c_{1}$ the solute concentration on the liquid side of the interface, and $\left.\partial_{\mathrm{n}} c\right|_{\mathrm{i}}$ the concentration gradient normal to the interface in the liquid. Additionally, interface equilibrium yields the Gibbs-Thomson condition

$$
T=T_{\mathrm{M}}+m c_{1}-\Gamma f(\vartheta) \kappa,
$$

where $T$ is the temperature, $T_{\mathrm{M}}$ is the melting temperature of the pure solvent, $m$ is the alloy liquidus slope, $\gamma_{0} f(\vartheta)=\gamma(\vartheta)+\mathbf{d}^{2} \gamma(\vartheta) / \mathrm{d} \vartheta^{2}$ is the interface stiffness where $\gamma(\vartheta)$ is the excess free energy of the solid-liquid interface dependent on the orientation $\vartheta$ and $\gamma_{0}$ is its value averaged over all orientations, $\quad \Gamma=\gamma_{0} T_{\mathrm{M}} / L$ is the Gibbs-Thomson coefficient where $L$ is the latent heat of fusion per unit volume, and $\kappa$ is the interface curvature.

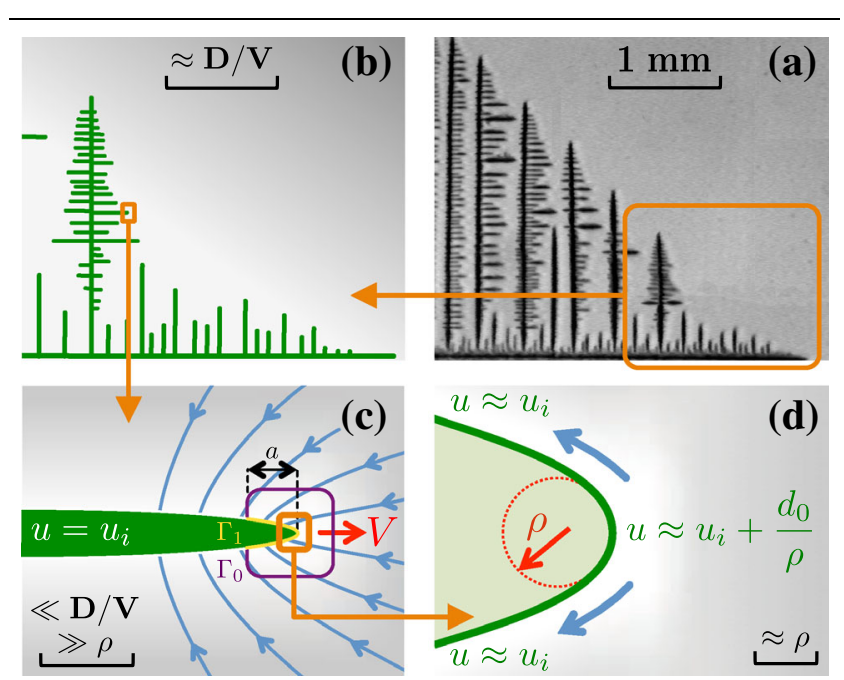

Fig. 1. The dendritic needle network model represents a dendritic grain, such as the ammonium bromide crystal from Ref. 33 in (a), as a hierarchical network of needle-like branches interacting through the long-range diffusion field, as illustrated in (b). The instantaneous tip radius $\rho(t)$ and velocity $V(t)$ of each branch are established by combining two conditions at distinct length scales: (c) a solute conservation condition at an intermediate scale larger than the tip radius $\rho$ but smaller than the diffusion length $D / V$, and (d) a solvability condition at the scale of the tip radius $\rho$. 
In constrained solidification within a given temperature gradient $G$ at a fixed growth velocity (or pulling velocity) $V_{\mathrm{p}}$ in the $x$ direction, the temperature field has the form

$$
T=T_{0}+G\left(x-V_{\mathrm{p}} t\right),
$$

where $T_{0}$ is a reference temperature, here chosen as the solidus temperature of the alloy, with its corresponding liquid equilibrium composition for a flat interface given by $c_{1}^{0}=\left(T_{\mathrm{M}}-T_{0}\right) /|m|=c_{\infty} / k$, where $c_{\infty}$ is the nominal composition of the alloy. Thus, the Gibbs-Thomson condition can be rewritten as

$$
\frac{c_{1}}{c_{1}^{0}}=1-(1-k) d_{0} f(\vartheta) \kappa-(1-k) \frac{x-V_{\mathrm{p}} t}{l_{\mathrm{T}}},
$$

where $d_{0} \equiv \Gamma /\left[|m|(1-k) c_{1}^{0}\right]$ is the capillarity length at $T_{0}$ and $l_{\mathrm{T}} \equiv\left[|m|(1-k) c_{1}^{0}\right] / G$ is the thermal length.

We introduce the reduced solute field

$$
u \equiv \frac{c_{1}^{0}-c}{(1-k) c_{1}^{0}} .
$$

The equilibrium value of $u$ on the liquid side of the interface is thus $u_{\mathrm{i}}=\left(c_{1}^{0}-c_{1}\right) /\left[(1-k) c_{1}^{0}\right]$, and the sharp-interface problem defined by the diffusion equation in the melt 1 , the mass conservation Stefan equation 2, and the equilibrium Gibbs-Thomson condition 3 summarizes to

$$
\begin{gathered}
\partial_{t} u=D \nabla^{2} u \\
{\left[1-(1-k) u_{\mathrm{i}}\right] v_{\mathrm{n}}=\left.D \partial_{\mathrm{n}} u\right|_{\mathrm{i}}} \\
u_{\mathrm{i}}=d_{0} f(\vartheta) \kappa+\frac{x-V_{\mathrm{p}} t}{l_{\mathrm{T}}},
\end{gathered}
$$

together with the far-field condition $c \rightarrow c_{\infty}$ as

$$
u(x \rightarrow+\infty)=1 .
$$

Then, to calculate the instantaneous growth velocity $V(t)$ and $\rho(t)$ of the tip of each dendritic branch, we combine two independent conditions at distinct length scales. Given the scale separation between the needle tip radius $\rho$ and the diffusion length $D / V$, one can write a mass conservation condition at an intermediate scale $\ll D / V$, such that diffusion is quasi-instantaneous, but $\gg \rho$, such that the needle appears like a very thin paraboloid of negligible curvature along its sides, as illustrated in Fig. 1c. We integrate the solute conservation at the interface, $D \partial u / \partial n \approx v_{\mathrm{n}}$, over a length $a$ between the tip location $x_{\mathrm{t}}$ and $x_{\mathrm{t}}-a$ (see Fig. 1c). If the needle tip has a circular cross-section of radius $r_{\mathrm{i}}(x)=\sqrt{2 \rho\left(x_{\mathrm{t}}-x\right)}$, the integration over a surface $\Gamma_{1}$ between $x=x_{\mathrm{t}}$ and $x=x_{\mathrm{t}}-a$ yields

$$
D \iint_{\Gamma_{1}} \frac{\partial u}{\partial n} \mathrm{~d} \Gamma_{1}=\int_{0}^{2 \pi} \int_{0}^{\mathrm{r}_{\mathrm{a}}} \mathrm{Vr} \mathrm{dr} \mathrm{d} \theta=2 \pi \mathrm{a} \rho \mathrm{V}
$$

with $r_{\mathrm{a}}=\sqrt{2 \rho a}$ the radius of the paraboloid at $x=x_{\mathrm{t}}-a$. Thus, the flux condition at the tip is

$$
\rho V=D F,
$$

where we defined the flux intensity factor

$$
F \equiv \frac{1}{2 \pi a} \iint_{\Gamma_{1}} \frac{\partial u}{\partial n} \mathrm{~d} \Gamma_{1} .
$$

To calculate $F$, we consider the Laplace equation in a moving frame of velocity $V$ around the tip,

$$
D \nabla^{2} u=-V \frac{\partial u}{\partial x} .
$$

Thus, if we integrate $\nabla^{2} u$ over a volume $\Sigma$ bounded by the surfaces $\Gamma_{0}$ and $\Gamma_{1}$, as shown in Fig. 1c, we can use the divergence theorem to write

$$
\begin{aligned}
\iint_{\Gamma_{1}} \frac{\partial u}{\partial n} \mathrm{~d} \Gamma_{1}-\iint_{\Gamma_{0}} \frac{\partial \mathrm{u}}{\partial \mathrm{n}} \mathrm{d} \Gamma_{0} & =-\iiint_{\Sigma} \nabla^{2} u \mathrm{~d} \Sigma \\
& =\frac{V}{D} \iiint_{\Sigma} \frac{\partial u}{\partial x} \mathrm{~d} \Sigma
\end{aligned}
$$

choosing a normal $n$ pointing outward of the closed contour $\left(\Gamma_{0}+\Gamma_{1}\right)$ along $\Gamma_{0}$, while pointing outward of the dendrite, i.e., inward the contour, along $\Gamma_{1}$-hence the minus sign in the left-hand side of Eq. 15. Thus, $F$ can be calculated from Eq. 13 where the integral along $\Gamma_{1}$ is given by

$$
\iint_{\Gamma_{1}} \frac{\partial u}{\partial n} \mathrm{~d} \Gamma_{1}=\iint_{\Gamma_{0}} \frac{\partial \mathrm{u}}{\partial \mathrm{n}} \mathrm{d} \Gamma_{0}+\frac{\mathrm{V}}{\mathrm{D}} \iiint_{\Sigma} \frac{\partial \mathrm{u}}{\partial \mathrm{x}} \mathrm{d} \Sigma,
$$

using any given surface $\Gamma_{0}$ intersecting the needle at $x=x_{\mathrm{t}}-a$, and its corresponding inner volume $\Sigma$.

We combine the first condition 12 with a solvability condition at the scale of the tip radius $\rho$, as illustrated in Fig. 1d. This condition for the existence of a growing shape-preserving paraboloid can be written as $^{35-37}$

$$
\rho^{2} V=\frac{1}{1-(1-k) u_{\mathrm{t}}} \frac{2 D d_{0}}{\sigma},
$$

where the first factor on the right-hand side stands for the temperature dependence of the capillarity length. With $d_{0}$ expressed at the reference (solidus) temperature $T_{0}$, the capillarity length at the tip location $d_{0}^{*}=d_{0} /\left[1-(1-k) u_{\mathrm{t}}\right]$ depends on the equilibrium concentration for a flat interface at the tip location $u_{\mathrm{t}} \cdot{ }^{15}$ Phase-field calculations have validated the steady-state solvability theory, ${ }^{38}$ and have also shown that $\rho^{2} V$ reaches a constant value dur- 
ing the early growth stage while both $\rho(t)$ and $V(t)$ exhibit significant variations in time, ${ }^{39}$ which allows us to use the relation 17 at all time in the model.

In summary, the three-dimensional DNN model for directional solidification is obtained by coupling the diffusion equation in the melt 7 with a network of needles at interfacial equilibrium, i.e., at $u=u_{\mathrm{i}} \approx\left(x-V_{\mathrm{p}} t\right) / l_{\mathrm{T}}$. The tip growth velocity $V(t)$ of each individual branch is obtained by combining Eqs. 12 and 17. While the product $\rho(t)^{2} V(t)$ is constant, with $\sigma$ set by the strength of interface freeenergy anisotropy, ${ }^{36}$ the product of $\rho(t) V(t)$ evolves in time like the flux intensity factor $F(t)$, given by Eqs. 13 and 16, which captures the long-range evolution of the solute field.

Additionally, as proposed in Ref. 15, sidebranches are generated periodically at a distance $d=$ $(N \pm \Delta N / 2) \rho$ behind a needle tip of radius $\rho$ each time the needle grows by a distance $d$, with an initial sidebranch length $l_{0}^{\mathrm{SB}}=\sqrt{2 \rho d}+\rho$. The frequency of sidebranching events $N$ and its fluctuation amplitude $\Delta N$ are input parameters to the model, chosen to match experimental observations, i.e., sidebranches appearing at a distance of five to ten tip radii behind the parent tip. ${ }^{33}$ We have shown that, as long as sidebranching is frequent enough for competition among branches to occur, the exact choice of the input parameters $N$ and $\Delta N$ has little influence on the final selected spacings. ${ }^{15}$

One limitation of the current implementation is that it does not describe the lateral movement of dendrites. Independent phase-field simulations show that the lateral drift velocity of dendrites induced by inhomogeneities of spacings within a well-oriented grain is orders of magnitude smaller than the solidification front velocity. Hence, this drift has a negligible effect on spacing selection in the present simulations, which is controlled by elimination and tertiary branching of well-oriented dendrites. However, in applications to polycrystalline growth competition, the current implementation does not describe the lateral movement of dendrites that is, for instance, responsible for the unusual overgrowth of favorably oriented dendrites at a converging grain boundary. ${ }^{31,32}$

\section{DIRECTIONAL SOLIDIFICATION EXPERIMENTS}

We benchmark the predictions of the model against well-controlled directional solidification experiments, combined with in situ x-ray radiography.

We prepared an aluminum-silicon alloy of nominal composition $c_{\infty}=9.8 \mathrm{wt}$. $\%$ Si by inductive melting of high-purity (99.999\%) $\mathrm{Al}$ and Si together in an argon atmosphere in a graphite crucible. The resulting ingot was homogenized at $575^{\circ} \mathrm{C}$ for $24 \mathrm{~h}$ and furnace cooled, then was surface-ground to remove the thin oxide, and warm-rolled to a thickness of $200 \mu \mathrm{m}$. Chemical analysis of the final foils using direct-current (DC) plasma emission spectroscopy (ASTM E 1097-12) confirmed a composition of $\mathrm{Al}-9.78 \mathrm{wt} . \% \mathrm{Si}$ with a trace amount of Zn (0.037 wt.\%). No other trace elements were found in excess of 0.003 wt.\%.

To proceed with the solidification experiments, we placed a sample in a boron nitride crucible that was inserted into a slotted steel rod with a hole normal to the x-ray beam direction. The steel rod was heated by two independent induction coils above and below the sample position and was equipped with a dozen thermocouples at different locations to provide measurement of the thermal conditions. We set and stabilized the temperature gradient in the rod with the temperature of the coils above (top) and below (bottom) the melting point of the alloy. We then reduced the power in the coils to let the solidification proceed from the bottom of the sample upward, using feedback control from the thermocouples to ensure a constant cooling rate. (Pictures and schematics of the experimental setup appear in the Supplementary Material.)

X-ray radiography images were captured in situ using a monochromatic synchrotron beam at $18 \mathrm{keV}$ at the Sector 32 Insertion Device beamline at Argonne National Laboratory's Advanced Photon Source. After passing through the sample, x-rays impinge upon a scintillator where they are converted to visible light, reflected by a mirror, and then captured with a camera that records a field of view of approximately $1.4 \mathrm{~mm} \times 1.7 \mathrm{~mm}$ in $1024 \times 1280$ pixel images, with a demagnified pixel size of $1.36 \mu \mathrm{m}$ and a frame rate of $3.54 \mathrm{~Hz}$.

We extracted dendrite tip velocities from image processing, and temperature gradients from thermocouple measurements. We compare the simulation results with three experiments, with a temperature gradient $G \approx 54 \pm 0.2 \mathrm{~K} / \mathrm{cm}$ and different growth velocities $V_{\mathrm{p}} \approx 40 \pm 8 \mu \mathrm{m} / \mathrm{s}, 106 \pm 20 \mu \mathrm{m} / \mathrm{s}$, and $285 \pm 50 \mu \mathrm{m} / \mathrm{s}$. Figure 2 shows an example x-ray image for $G \approx 54 \mathrm{~K} /$ $\mathrm{cm}$ and $V_{\mathrm{p}} \approx 285 \mu \mathrm{m} / \mathrm{s}$.

\section{SIMULATIONS}

We investigated the dynamical selection of primary dendritic spacings during the directional solidification experiments described in the previous

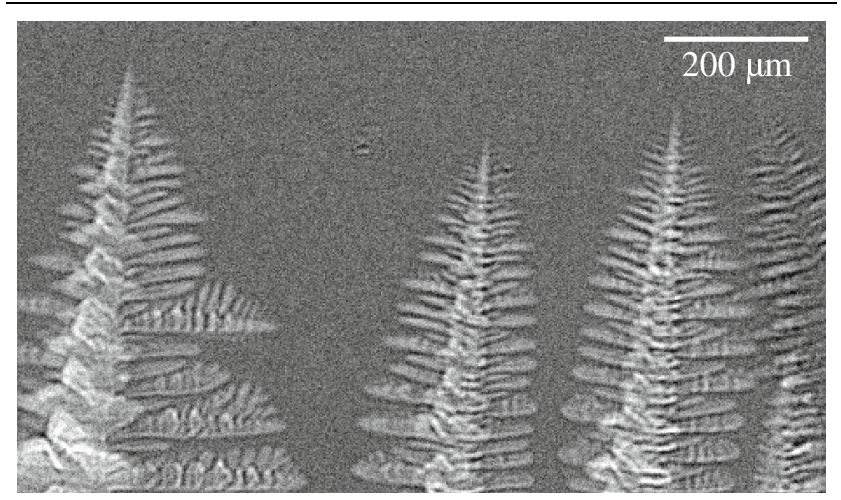

Fig. 2. X-ray radiography image of an Al-9.8 wt.\%Si directional solidification experiment, for a temperature gradient $G \approx 54 \mathrm{~K} / \mathrm{cm}$ and a growth velocity $V_{\mathrm{p}} \approx 285 \mu \mathrm{m} / \mathrm{s}$. 
section using three-dimensional DNN simulations. Simulations were discretized using a finite-difference cubic grid of uniform mesh size $\Delta x$, and an explicit Euler time scheme with a time step $\Delta t \approx \Delta x^{2} /(6 D)$. The model was implemented on graphics processing units (GPUs) using the Compute Unified Device Architecture (CUDA). Each simulation was achieved in single precision on a single GPU. We used the alloy, process, and numerical parameters listed in Table I. Alloy parameters were taken from the literature. ${ }^{40,41}$ From Ref. 36, the tip selection parameter corresponding to an interfacial anisotropy strength $\epsilon_{4}=0.012^{42}$ is $\sigma \approx 0.058$ for a one-sided model in $3 \mathrm{D}$. We used a finite-difference grid spacing between 1.6 and 2.1 times the analytical steady-state tip radius $\rho_{\mathrm{s}}$, calculated using microscopic solvability together with the Ivantsov solution. ${ }^{41,43}$ During the simulations, we tracked the position of the most advanced tip and shifted the domain periodically to keep this position fixed in the simulation box. To prevent numerical instabilities due to the constancy of $\rho^{2} V$ when a needle is stopped and its tip velocity $V$ decreases to zero, we bounded the thickness of the needles to $3 \rho_{\mathrm{s}} \cdot{ }^{34}$ At the tip of each needle, the integration surface $\Gamma_{0}$ for the evaluation of $F(t)$ spanned three grid points behind and three grid points ahead of the tip location in the direction of the needle growth, and in the two directions normal to the needle growth it was one grid point wider than the thickness of the needle at its intersection with $\Gamma_{0}$. All boundary conditions were set to symmetric, i.e., no solute flux through the domain boundaries. The size of the simulation in $z$ was $100 \mu \mathrm{m}$, to simulate one half of the experimental sample thickness.

We performed three series of simulations. The first series aimed at characterizing the primary spacing stability range for the velocity range relevant to the experiments. To do so, we proceeded similarly as in Ref. 15. For a given set of conditions, we started a simulation with two needles located along the opposite $y$ boundaries of the domain, and repeated the simulation with different sizes of the domain in the $y$ direction, thus testing the stability of two primary branches at a given initial spacing. If the $y$-dimension (i.e., the initial spacing) is too low, one of the two primary branches is eliminated and only one primary needle and its sidebranches remain at the end of the simulation. If the initial spacing is stable, the two primary needles still coexist in the steady state. If the initial spacing is too high, one of the two primary trunks will be at the origin of a tertiary sidebranch that will stabilize as a new primary dendrite between the two initial dendrites. We performed those simulations for the three experimental growth velocities $(40 \mu \mathrm{m} / \mathrm{s}$, $106 \mu \mathrm{m} / \mathrm{s}$, and $285 \mu \mathrm{m} / \mathrm{s}$ ), as well as three intermediate velocities $(75 \mu \mathrm{m} / \mathrm{s}, 175 \mu \mathrm{m} / \mathrm{s}$, and $255 \mu \mathrm{m} / \mathrm{s})$. Sorted by increasing growth velocity, the simulation sizes in the growth direction $x$ were $5780 \mu \mathrm{m}$,
Table I. Simulation parameters

\begin{tabular}{|c|c|c|c|}
\hline Symbol & Value & Unit & \\
\hline$c_{\infty}$ & 9.8 & wt.\%Si & \\
\hline$m$ & -6.5 & $\mathrm{~K} / \mathrm{wt} . \% \mathrm{Si}$ & 40 \\
\hline$D$ & $3 \times 10^{-9}$ & $\mathrm{~m}^{2} / \mathrm{s}$ & 41 \\
\hline$\Gamma$ & $1.96 \times 10^{-7}$ & $\mathrm{Km}$ & 40 \\
\hline$k$ & 0.13 & & 40 \\
\hline$\sigma$ & 0.058 & & \\
\hline$G$ & 54 & $\mathrm{~K} / \mathrm{cm}$ & \\
\hline$V_{\mathrm{p}}$ & $\begin{array}{c}40,75,106 \\
175,225,285\end{array}$ & $\mu \mathrm{m} / \mathrm{s}$ & \\
\hline$\Delta x$ & $\begin{array}{l}1.62,1.88,1.94 \\
1.97,1.84,2.08\end{array}$ & $\rho_{\mathrm{s}}$ & \\
\hline$\Delta x$ & $\begin{array}{l}4.76,4.0,3.45 \\
2.70,2.22,2.22\end{array}$ & $\mu \mathrm{m}$ & \\
\hline$\Delta N$ & 10 & & \\
\hline$\vec{N}$ & 15 & & \\
\hline
\end{tabular}

$4080 \mu \mathrm{m}, \quad 2310 \mu \mathrm{m}, \quad 1720 \mu \mathrm{m}, \quad 1420 \mu \mathrm{m}$, and $1380 \mu \mathrm{m}$, and the simulated times were $300 \mathrm{~s}$, $240 \mathrm{~s}, 180 \mathrm{~s}, 140 \mathrm{~s}, 100 \mathrm{~s}$, and $60 \mathrm{~s}$. As for all simulations in this paper, we started with primary dendrites within the $(z=0)$ plane, with their tips located at the liquidus temperature.

The second series of simulations investigated the dynamical selection of spacing in spatially extended dendrite arrays. Similarly as in 2D in Ref. 15, we simulated primary spacing selection by elimination/coarsening after the destabilization of a planar front, by starting a simulation with several primary needles evenly spaced in the $y$ direction. We simulated the three experimental growth velocities $V=40 \mu \mathrm{m} / \mathrm{s}, 106 \mu \mathrm{m} / \mathrm{s}$, and $285 \mu \mathrm{m} / \mathrm{s}$, respectively, using domains of $(x \times y)$ dimensions (5471 $\times$ $\left.5014 \mu \mathrm{m}^{2}\right), \quad\left(2086 \times 3134 \mu \mathrm{m}^{2}\right), \quad$ and $(1344 \times$ $2020 \mu \mathrm{m}^{2}$ ), respectively, initialized with 118,102 , and 102 needles. We applied a random perturbation of amplitude $10^{-3} \Delta x$ on the initial size of the needles to break the initial symmetry of the problem and prevent numerical rounding errors as the only origin of the planar front destabilization. ${ }^{15}$ For similar velocities and domain sizes, we also investigated the spacing selection by tertiary branching, as in Ref. 15, with a simulation starting as one steady-state primary dendrite growing within $1 / 10$ of the total $y$ dimension, and abruptly increasing the cross-section to the whole size in $y$.

The third and final series of simulations explored the influence of the mold geometry on the primary spacing selection by branching. Similarly as in the case of a sudden cross-section increase, we started the simulation with one primary dendrite growing in a channel of $1 / 10$ of the total $y$-dimension. The cross-section in the $y$ direction then increased linearly, simulating a wedge-shaped mold with sides at an angle $\theta$ with the thermal gradient direction, as schematized in Fig. 3. (The case of a sudden crosssection widening hence corresponds to the limit 


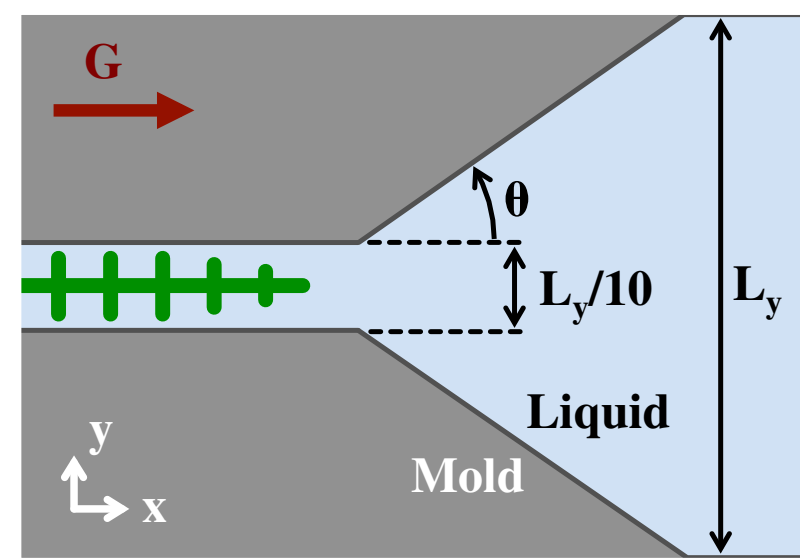

Fig. 3. Schematics of the wedge geometry. A single dendrite initially grows in a narrow channel 10 times smaller than the total size of the domain $L_{y}$, which opens up to the final $y$-size with an angle $\theta$.

$\theta=90^{\circ}$.) In the code, the sides of the wedge were implemented as steps, composed of no-flux boundary conditions along $x$ and $y$. (Details and schematics of the boundary conditions appear in the Supplementary Material.) For a velocity $V_{\mathrm{p}}=106 \mu \mathrm{m} / \mathrm{s}$ and a size in $(x \times y)$ of $(2086 \times 2031) \mu \mathrm{m}^{2}$, we explored values of $\tan (\theta)=0.1,0.2,0.3,0.5,0.7,1,1.5,2.5,5$, and 10 .

To illustrate how multiscale these DNN simulations are, for the three experimental growth velocities, Table II lists the main length scales of the problem, namely the capillarity length at the steady-state tip location $d_{0}^{*}$, the steady-state dendritic tip radius $\rho_{\mathrm{s}}$, the diffusion length $D / V_{\mathrm{p}}$, and the thermal length $l_{\mathrm{T}}$, spanning seven orders of magnitude.

\section{RESULTS AND DISCUSSION}

First, we discuss the DNN predictions of stable spacing $\Lambda$ versus growth velocity $V_{\mathrm{p}}$. In Fig. 4 , small grey symbols represent the first series of simulations starting with two needles: squares show stable spacings, down-pointing triangles show initial spacings resulting in the elimination of one of the initial dendrites, and up-pointing triangles show simulations resulting in tertiary branching events with creation of a new primary dendrite. For clarity, only a few of these simulations are reported in Fig. 4, as the lower (elimination) limit $\Lambda_{\min }$ and an upper (branching) limit $\Lambda_{\max }$ are estimated up to a precision of $\pm \Delta x$. The stability limits are fit to power laws $\Lambda \sim V^{-\alpha}$, and plotted in Fig. 4 as solid lines with $\alpha=0.68$ and 0.53 , respectively, for $\Lambda_{\min }$ and $\Lambda_{\max }$. Both limits are relatively well approximated by $\Lambda \sim V^{-1 / 2}$, in black dotted lines in Fig. 4 . In contrast to the assumption that the ratio $\Lambda_{\max } / \Lambda_{\min }$ is close to 2 or 3 (see, e.g., Ref. 12), we find a ratio of 4 between prefactors of the fits to $V^{-1 / 2}$ (exactly 3.97 ), in agreement with quantitative phase-field simulations for a succinonitrile-salol alloy. ${ }^{14}$
Experimental measurements of primary spacings from x-ray imaging appear as green circles in Fig. 4. The measurements fall within the predicted stable range. However, they are close to the upper limit $\Lambda_{\text {max }}$. In comparison, Fig. 4 shows the spacings observed for the second series of spatially extended DNN simulations, which are mostly within the lower half of the stability range, similarly as in $2 \mathrm{D},{ }^{15}$ for both the case of a planar interface destabilization (Fig. 5a) and the case of a sudden crosssection widening (Fig. 5e). As seen in Fig. 4, the highest spacings observed in the simulations among about 15 spacings are relatively close to experimental measurements. To ensure numerical convergence with the grid spacing $\Delta x$, we carried out similar simulations with $\Delta x \approx \rho_{\mathrm{s}}$, i.e., with a grid spacing twice smaller, which yielded similar primary spacing selection.

The discrepancy between simulations and experiments may be in part attributed to the uncertainty in the selection parameter $\sigma$ for Al-Si alloys. Its value depends upon interface anisotropy that has only been estimated for an $\mathrm{Al}-\mathrm{Cu}$ alloy, ${ }^{44}$ which could differ for Al-Si. The value used in the current simulations derives from atomistic simulations for pure $\mathrm{Al},{ }^{42}$ combined with linear solvability theory. ${ }^{36}$ Exploratory simulations for planar destabilization and cross-section widening with a lower $\sigma=0.02$ at $V=106 \mu \mathrm{m} / \mathrm{s}$ yielded a much broader spacing range from $150 \mu \mathrm{m}$ to $530 \mu \mathrm{m}$, which includes the experimental point of Fig. 4. Hence, the value of the selection parameter $\sigma$ for Al-Si alloys warrants further investigation, to reach a fully quantitative comparison of modeling with experiments. In comparison, DNN simulations compared with measurements during directional solidification of an $\mathrm{Al}-$ $\mathrm{Cu}$ alloy led to much better agreement. ${ }^{34}$

Other possible sources of discrepancy between simulations and experiments include: the limited sample size of the measurements (e.g., only two spacings in Fig. 2), solutal convection (observed in Al-Cu thin samples ${ }^{45}$ ), the development of a solute boundary layer during the thermal stabilization of the melted sample, ${ }^{46}$ the thermal inertia of the setup at the initiation of cooling, ${ }^{47}$ as well as uncertainties in materials parameters and processing conditions.

Interestingly, spacing selection after planar destabilization and cross-section widening yielded similar spacings, unlike in $2 \mathrm{D}$ where both $\mathrm{DNN}^{15}$ and phase-field ${ }^{16}$ systematically predict larger spacings from branching than from planar destabilization. This could be a dimensionality effect, as the solute field around a dendrite is very different in $2 \mathrm{D}$ and $3 \mathrm{D}$, with the constraint of in-plane solute redistribution in $2 \mathrm{D}$ perhaps leading to a tertiary branching inhibition, thus yielding a higher $\Lambda_{\max }$. These observations warrant further investigation.

In simulated arrays, some spacings are slightly lower than the elimination limit $\Lambda_{\min }$ (Fig. 4). This is due to the inhomogeneity of spacings throughout 


\section{Table II. Length scales in the DNN simulations}

\begin{tabular}{lcccc}
\hline Growth velocity, $V$ & 40 & 106 & 285 & $\mu \mathrm{m} / \mathrm{s}$ \\
Capillarity length, $d_{0}^{*}$ & 3.3 & 3.2 & 3.1 & $\mathrm{~nm}$ \\
Dendrite tip radius, $\rho_{\mathrm{s}}$ & 2.9 & 1.8 & 1.1 & $\mu \mathrm{m}$ \\
Diffusion length, $D / V_{\mathrm{p}}$ & 75 & 28 & 11 & $\mu \mathrm{m}$ \\
Thermal length, $l_{\mathrm{T}}$ & 79 & 79 & 79 & $\mathrm{~mm}$ \\
\hline
\end{tabular}

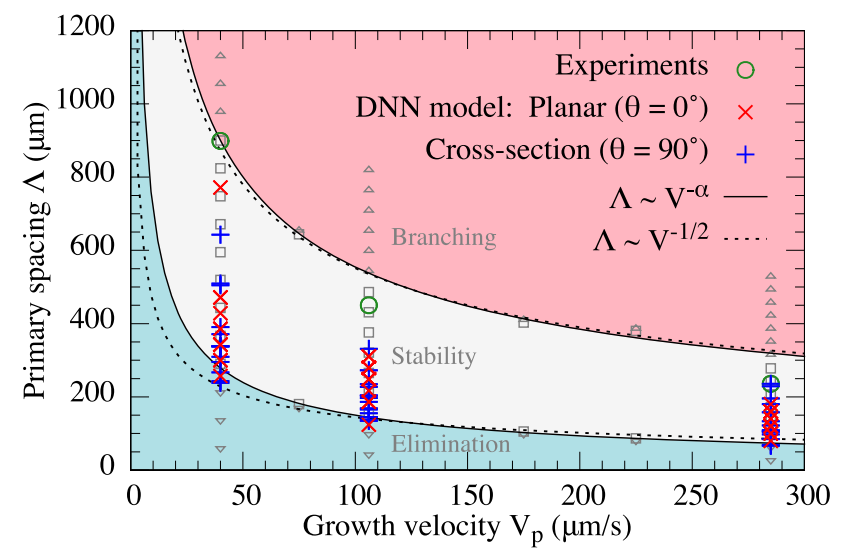

Fig. 4. Primary dendritic spacing versus growth velocity. Grey symbols represent simulations identifying the stable spacing range, i.e., starting with two primary dendrites until elimination instability $(\nabla)$, branching instability $(\triangle)$, or steady-state stability $(\square)$. The predicted spacings after a planar front destabilization $(\times$, see Fig. 5a), and sudden cross-section widening (+, see Fig. 5e) show individual steady-state spacings. Experimental measurements (o) show the average spacing for each velocity.

the sample. In Fig. 6, we illustrate how the asymmetrical solute distribution around dendrite tips can lead to a spacing between two dendrites $\Lambda<\Lambda_{\min }$, if the spacings on the outer sides of the two dendrites are sufficiently higher than $\Lambda_{\min }$. If we calculate an average spacing on the two sides of each dendrite, it falls within the stable limits of Fig. 4. While the current implementation of the model prevents the lateral drift of primary dendritic trunks, in experiments or phase-field simulations dendrites would slowly drift to evolve toward a homogeneous distribution of spacings.

The final series of simulations explored the influence of geometry on spacing, by changing the rate of widening of the sample cross-section at $V_{\mathrm{p}}=106 \mu \mathrm{m} / \mathrm{s}$. The results of these simulations are reported in Fig. 7, with simulations for $\tan (\theta)=0.1$, 1 , and 10 also illustrated in Fig. 5b-d. Figure 7 does not exhibit any conclusive $\theta$-dependence of the selected primary spacings. This is in agreement with $2 \mathrm{D}$ phase-field simulations that suggested that the primary spacing selection within a grain is not affected by the orientation of the boundary with the neighboring grain. ${ }^{16}$ These results show that solidification conditions-i.e., cooling rate, growth velocity, thermal gradient-have a more crucial influence over spacing selection than the geometry of the mold. Of course, unless thermal conditions are (a) Planar $\left(\theta=0^{\circ}\right)$
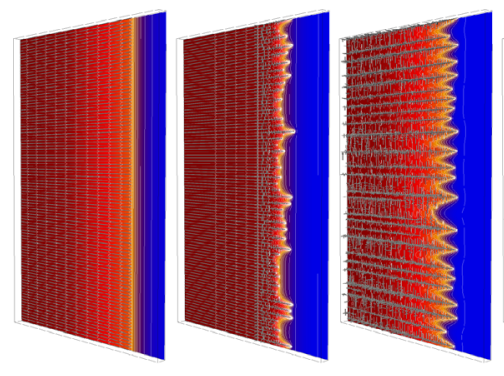

(b) Wedge $(\tan \theta=0.1)$
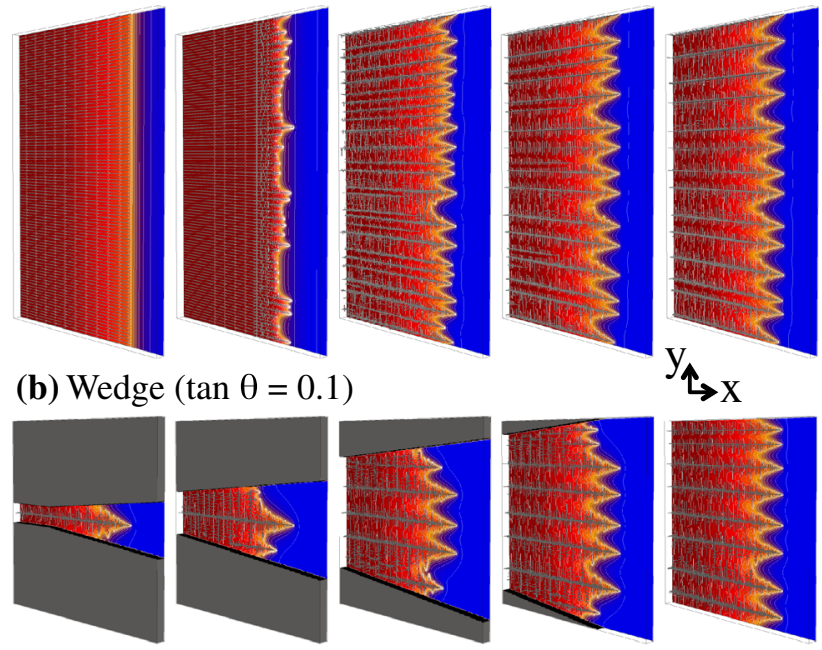

(c) Wedge $(\tan \theta=1)$
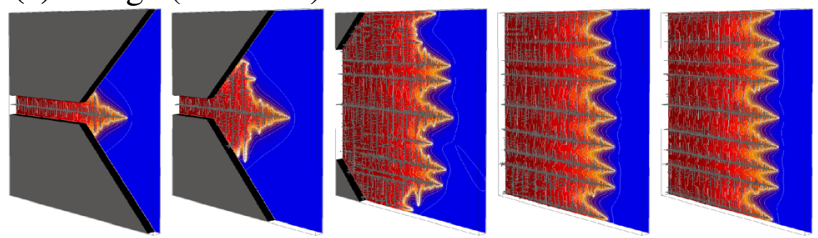

(d) Wedge $(\tan \theta=10)$
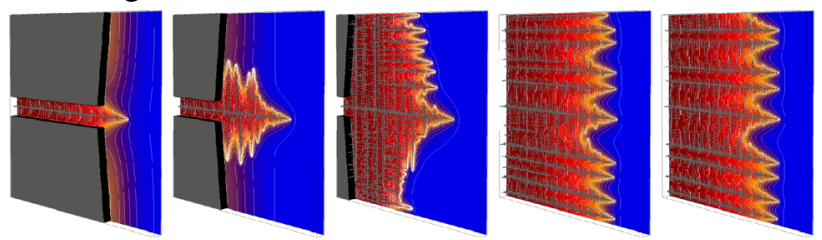

(e) $\operatorname{Cross}-\operatorname{section}\left(\theta=90^{\circ}\right)$
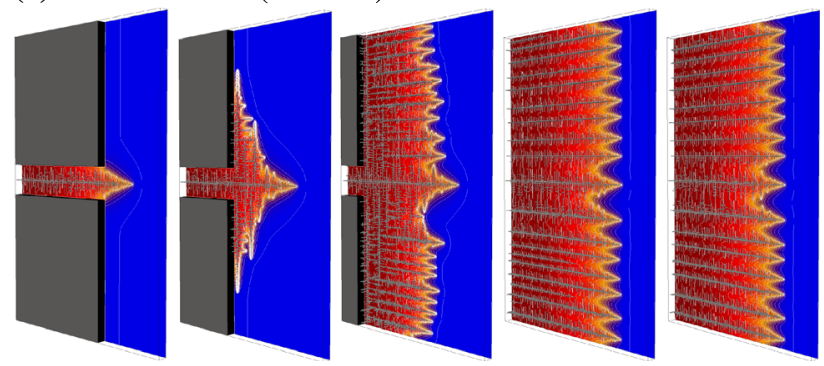

Fig. 5. Spacing selection during directional solidification at $V=106 \mu \mathrm{m} / \mathrm{s}$ for different geometries: (a) destabilization of a planar front; (b-d) progressive cross-section widening with a wedge angle $\theta$ for $\tan (\theta)=$ (b) 0.1 , (c) 1 , and (d) 10; (e) abrupt cross-section widening. Videos of (a), (c), and (e) are attached as Supplementary Material.

constrained, the geometry of a casting mold has a crucial influence on those solidification conditions, which is usually why wedge-shaped molds are used to study microstructure selection in the presence of a gradient of thermal conditions. These results suggest that an indirect coupling between thermal modeling of a cast ingot and local modeling of microstructure selection is a valid approach for spacing selection (see examples of such couplings with phase-field in Refs. 48,49). However, this type of 
(a) $\mathrm{V}=40 \mu \mathrm{m} / \mathrm{s}$

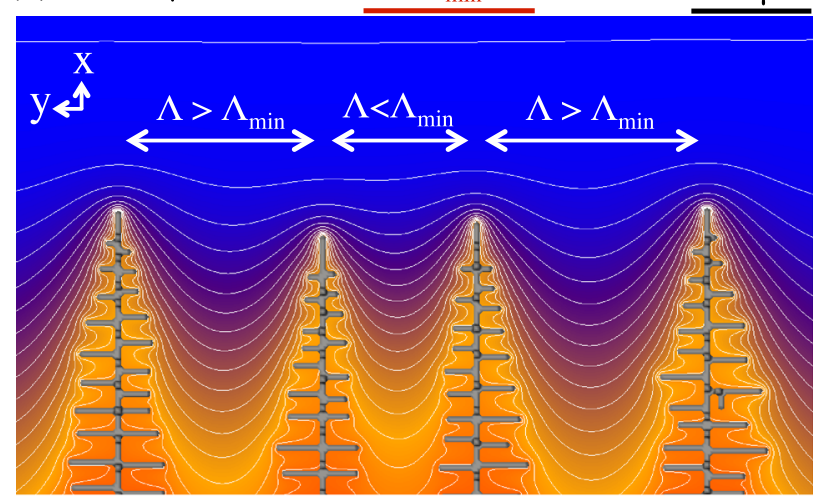

(b) $\mathrm{V}=106 \mu \mathrm{m} / \mathrm{s}$

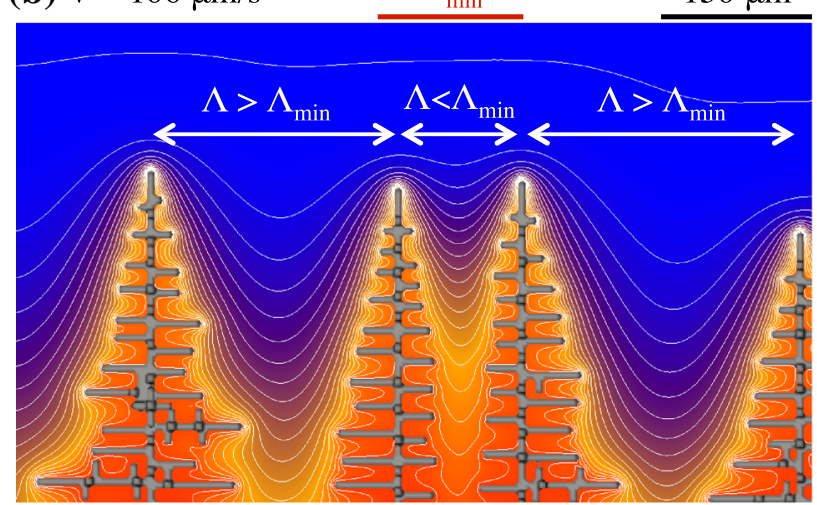

(c) $\mathrm{V}=285 \mu \mathrm{m} / \mathrm{s}$

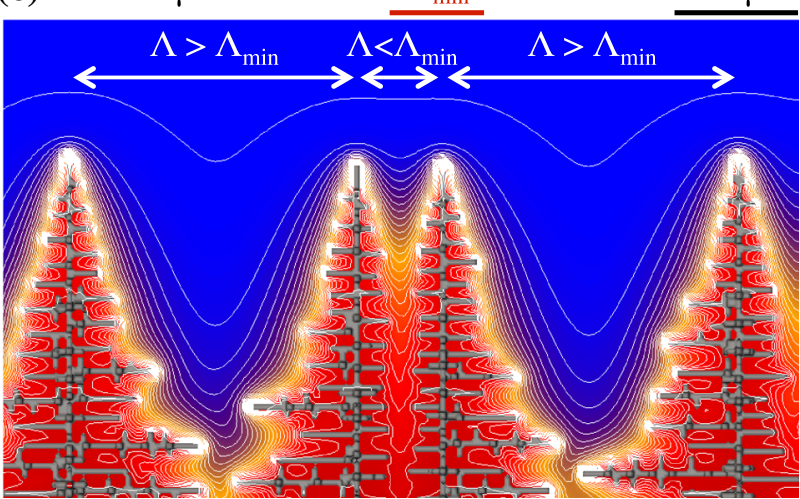

Fig. 6. Asymmetrical solute fields enabling stable primary spacings $\Lambda$ lower than the elimination limit $\Lambda_{\min }$, illustrated for the planar destabilization simulations at (a) $V=40 \mu \mathrm{m} / \mathrm{s}$ and (b) $106 \mu \mathrm{m} / \mathrm{s}$ and the cross-section widening case at (c) $V=285 \mu \mathrm{m} / \mathrm{s}$. The color map represents the $u(x, y)$ field in the $(z=0)$ plane, with white contour lines from $u=1$ (top) with downward steps of 0.001 .

coupling does not account for the history-dependent selection of spacing.

Additionally, as can be seen in Fig. 5c-e, simulations at high $\theta$ yield a spacing distribution with spacings at to the center of the domain in $y$ (i.e., close to the initial dendrite) among the largest in the final array. This is illustrated in Fig. 8, where we plot the average spacing around each dendrite $\Lambda$, i.e., the average of the two spacings on the two sides of a dendrite, as a function of its location in $y$ for six

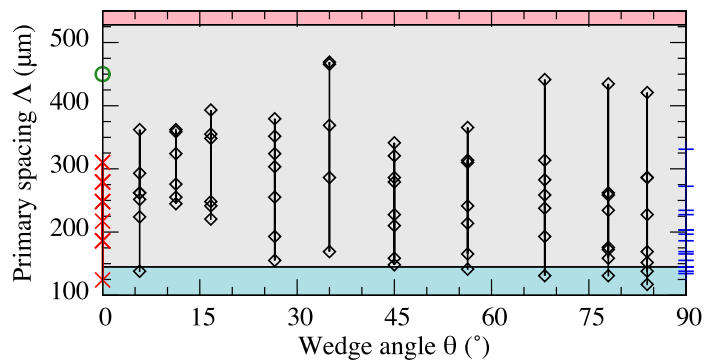

Fig. 7. Selected primary spacings versus wedge angle $\theta$, related to the cross-section widening rate. Symbols are similar to Fig. 4 , with additional $\diamond$ symbols for $0^{\circ}<\theta<90^{\circ}$ with $\tan (\theta)=0.1,0.2,0.3,0.5$, $0.7,1,1.5,2.5,5$, and 10 .

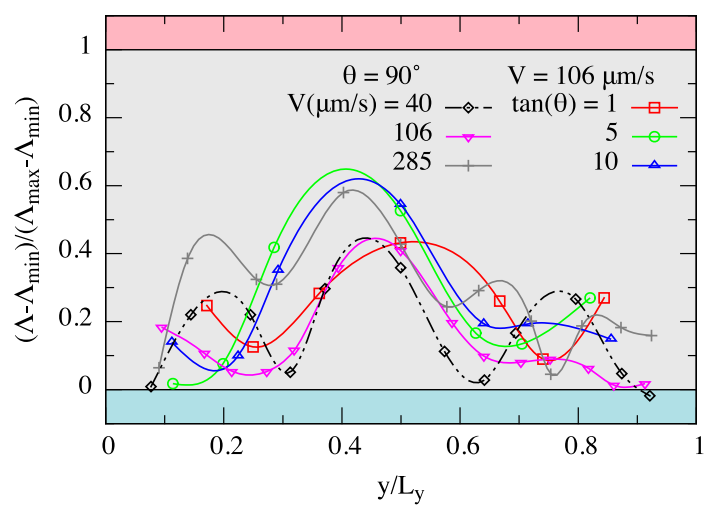

Fig. 8. Distribution of average spacing around dendrites along the $y$ axis for simulations at high $\theta$.

different simulations. Since we are comparing different velocities and domain sizes, the spacings are scaled between the calculated stability limits $\Lambda_{\text {min }}$ and $\Lambda_{\max }$, and the $y$-positions are scaled with respect to $L_{y}$, the total size of the domain in $y$. The larger spacings at the center of the domain are linked to the dynamics of the secondary branches at the origin of new tertiary/primary branches, first accelerating rapidly and then decelerating when tips reach the sides of the domain. The size of the arrays in the present simulations is not large enough to draw quantitative conclusions on spacing distributions among the array, and this topic still warrants deeper investigation. However, this illustrates how the DNN method can produce insight into spacing distribution in cast parts.

Finally, since the model was developed to overcome phase-field computational limitations for simulating concentrated alloys, it seems worth commenting on the computational efficiency of the simulations presented in this paper. First, the largest simulations presented here require less than $600 \mathrm{MB}$ of RAM, which makes them achievable with almost any GPU released in the past few years. All simulations were achieved on one Nvidia GTX680, except for the planar destabilization runs performed on an Nvidia GTX TITAN. For the first series of simulations, pertaining to the spacing stability 
range, each simulation took between $45 \mathrm{~min}$ (for $1380 \times 30 \times 100 \mu \mathrm{m}^{3}$ over $60 \mathrm{~s}$ at $\left.V=285 \mu \mathrm{m} / \mathrm{s}\right)$ and $140 \mathrm{~min}$ (for $5776 \times 1129 \times 100 \mu \mathrm{m}^{3}$ over $300 \mathrm{~s}$ at $V=40 \mu \mathrm{m} / \mathrm{s}$ ). Simulations of the second series with spatially extended domains took up to $17.5 \mathrm{~h}$ (for a domain of $1.3 \times 2.0 \times 0.1 \mathrm{~mm}^{3}$ over $3 \mathrm{~min}$ at $V=285 \mu \mathrm{m} / \mathrm{s}$ ). Simulations of the third series, for a domain of $2.1 \times 2.0 \times 0.1 \mathrm{~mm}^{3}$ over $9 \mathrm{~min}$ at $V=106 \mu \mathrm{m} / \mathrm{s}$, lasted between $8 \mathrm{~h}$ and $12 \mathrm{~h}$. The current study is thus fairly reasonable in terms of computational resources. A similar study with quantitative phase-field simulations would have been extremely challenging and computationally demanding, if feasible at all. Therefore, without replacing any of the existing models, we believe that the DNN model completes the palette of available tools, and paves the way for new studies of fundamental microstructure selection mechanisms in dendritic solidification at an intermediate scale between phase-field and volume-averaged approaches.

\section{CONCLUSION}

We have presented a three-dimensional version of the DNN model for directional solidification. Using the model, we have predicted the stable range of primary dendritic spacings for Al-9.8wt.\% Si alloy over a range of growth velocities $(40 \mu \mathrm{m} / \mathrm{s}$ to $285 \mu \mathrm{m} / \mathrm{s}$ ), and explored the dynamical selection of primary spacings for spatially extended dendritic arrays for different conditions, namely after a planar front destabilization and during a change of sample cross-section.

The spacings measured from in situ x-ray imaging of directional solidification experiments fall within the stable range predicted by the simulations. However, simulated spacings in spatially extended arrays were found to be smaller than the measured spacings. Preliminary simulations suggest that a lower value of the tip selection parameter $\sigma$, which still needs to be identified for Al-Si alloys, yields a broader distribution of spacings that include the experimental data point for $V=106 \mu \mathrm{m} /$ $\mathrm{s}$. This is in agreement with the expected trend of a small $\sigma$ leading to a larger tip radius for the same growth velocity, hence yielding a larger spacing. These findings highlight the importance of accurately determining $\sigma$, to reach fully quantitative predictions of microstructure selection, while illustrating the potential of the DNN modeling approach to provide computationally efficient simulations of the effect of $\sigma$ at the scale of one to several grains.

We did not observe any influence of the rate of cross-section widening on the selected spacings. In contrast to previous $2 \mathrm{D}$ simulations, ${ }^{15,16}$ we did not observe a systematic difference in primary spacings selection from elimination/coarsening (i.e., after a planar front destabilization) and from tertiary branching. These first three-dimensional applications of the DNN model also illustrate the potential of the approach to predict spatial distributions of spacings in a spatially extended sample.

The outlooks of this work are manyfold. They include polycrystalline implementations of the model that account for different grain orientations and the lateral drift of dendrites, and further coupling with additional physics, such as convective transport in the melt. We expect the DNN model and its extensions to yield deeper insight into microstructure selection at an intermediate scale between phasefield and volume-averaged grain dynamics models, for instance, exploring the fundamental mechanisms of polycrystalline grain growth competition, ${ }^{16}$ the influence of convective transport at the scale of extended dendritic arrays, ${ }^{50}$ or the columnar-to-equiaxed transition. ${ }^{51}$

\section{ACKNOWLEDGEMENTS}

D.T., A.J.C., S.D.I., P.J.G., and J.W.G. were supported by A.J.C.'s Early Career award from the U.S. Department of Energy, Office of Basic Energy Sciences, Division of Materials Sciences and Engineering and Los Alamos National Laboratory, operated by Los Alamos National Security, L.L.C. under Contract No. DE-AC52-06NA25396 for the U.S. Department of Energy. A.K. acknowledges support of Grant No. DE-FG02-07ER46400 from the U.S. Department of Energy, Office of Basic Energy Sciences. Use of the Advanced Photon Source, an Office of Science User Facility operated for the U.S. D.O.E. Office of Science by Argonne National Laboratory, was supported by the U.S. D.O.E. under Contract No. DE-AC02-06CH11357.

\section{REFERENCES}

1. M.C. Flemings, Solidification Processing (McGraw-Hill, New York, 1974).

2. R.E. Jesse and H. Giller, J. Cryst. Growth 7, 348-352 (1970).

3. J.D. Hunt, Solidification and Casting of Metals (The Metals Society, London, 1979), pp. 3-9.

4. W. Kurz and D.J. Fisher, Acta Metall. 29, 11-20 (1981).

5. K. Somboonsuk, J.T. Mason, and R. Trivedi, Metall. Trans. 15A, 967-975 (1984).

6. K. Somboonsuk and R. Trivedi, Acta Metall. 33, 1051-1060 (1985).

7. R. Trivedi and K. Somboonsuk, Acta Metall. 33, 1061-1068 (1985).

8. W. Huang, X. Geng, and Y. Zhou, J. Cryst. Growth 134, 105115 (1993)

9. S.H. Han and R. Trivedi, Acta Metall. Mater. 42, 25-41 (1994).

10. J. Bechhoefer and A. Libchaber, Phys. Rev. B 35, 1393 (1987).

11. J.A. Warren and J.S. Langer, Phys. Rev. E 47, 2702 (1993).

12. D. Ma, Metall. Mater. Trans. B 35B, 735-742 (2004).

13. C.-A. Gandin, M. Eshelman and R. Trivedi, Metall. Mater. Trans. A 27A, 2727-2739 (1996).

14. B. Echebarria, A. Karma and S. Gurevich, Phys. Rev. E 81, 021608 (2010).

15. D. Tourret and A. Karma, Acta Mater. 61, 6474-6491 (2013).

16. D. Tourret and A. Karma, Acta Mater. 82, 64-83 (2015).

17. C.-A. Gandin and M. Rappaz, Acta Mater. 45, 2187-2195 (1997).

18. C.-A. Gandin, J. Desbiolles, M. Rappaz, and P. Thévoz, Metall. Mater. Trans. A 30A, 3153-3165 (1999). 
19. C.-A. Gandin, T. Carozzani, H. Digonnet, S. Chen, and G. Guillemot, JOM 65, 1122-1130 (2013).

20. W.J. Boettinger, J.A. Warren, C. Beckermann, and A. Karma, Annu. Rev. Mater. Res. 32, 163-194 (2002).

21. A. Karma and W.J. Rappel, Phys. Rev. E 57, 4323 (1998).

22. A. Karma, Phys. Rev. Lett. 87, 115701 (2001).

23. N. Provatas, M. Greenwood, B. Athreya, N. Goldenfeld, and J. Dantzig, Int. J. Mod. Phys. B 19, 4525-4565 (2005).

24. W.L. George and J.A. Warren, J. Comput. Phys. 177, 264283 (2002)

25. T. Shimokawabe, T. Aoki, T. Takaki, A. Yamanaka, A. Nukada, T. Endo, N. Maruyama, and S. Matsuoka: Proceedings of SC11, 1-11 (2011).

26. N. Bergeon, D. Tourret, L. Chen, J.-M. Debierre, R. Guérin, A. Ramirez, B. Billia, A. Karma, and R. Trivedi, Phys. Rev. Lett. 110, 226102 (2013).

27. S. Gurevich, A. Karma, M. Plapp, and R. Trivedi, Phys. Rev. E 81, 011603 (2010).

28. Z. Wang, J. Li, J. Wang, and Y. Zhou, Acta Mater. 60, 1957$1964(2012)$

29. M. Amoorezaei, S. Gurevich, and N. Provatas, Acta Mater. 58, 6115-6124 (2010).

30. S. Gurevich, M. Amoorezaei, and N. Provatas, Phys. Rev. E 82, 051606 (2010).

31. J. Li, Z. Wang, Y. Wang, and J. Wang, Acta Mater. 60, 14781493 (2012)

32. T. Takaki, M. Ohno, T. Shimokawabe, and T. Aoki, Acta Mater. 81, 272-283 (2014).

33. Y. Couder, J. Maurer, R. González-Cinca, and A. HernándezMachado, Phys. Rev. E 71, 031602 (2005).

34. D. Tourret, A. Karma, A.J. Clarke, P.J. Gibbs, and S.D. Imhoff, Proceedings of the 14th International Conference on
Modeling of Casting, Welding, and Advanced Solidification Processes (MCWASP XIV), accepted (2015).

35. J.S. Langer, Chance and Matter, Lectures on the Theory of Pattern Formation, Les Houches XLVI, 629-711 (1987).

36. A. Barbieri and J.S. Langer, Phys. Rev. A 39, 5314 (1989).

37. M. Ben Amar and E. Brener, Phys. Rev. Lett. 71, 589 (1993).

38. A. Karma and W.-J. Rappel, J. Cryst. Growth 174, 54-64 (1997).

39. M. Plapp and A. Karma, Phys. Rev. Lett. 84, 1740 (2000).

40. C.-A. Gandin, Acta Mater. 48, 2483-2501 (2000).

41. W. Kurz and D.J. Fisher, Fundamentals of Solidification, 3rd edn. (Trans. Tech. Pub, Aedermannsdorf, 1992).

42. J.R. Morris, Phys. Rev. B 66, 144104 (2002).

43. G.P. Ivantsov, Dokl. Akad. Nauk. SSSR 58, 567-569 (1947).

44. R.E. Napolitano, S. Liu, and R. Trivedi, Interface Sci. 10, 217-232 (2002).

45. A. Bogno, H. Nguyen-Thi, A. Buffet, G. Reinhart, B. Billia, N. Mangelinck-Noël, N. Bergeon, J. Baruchel, and T. Schenk, Acta Mater. 59, 4356-4365 (2011).

46. M. Xu, L.M. Fabietti, Y. Song, D. Tourret, A. Karma, and R. Trivedi, Scripta Mater. 88, 29-32 (2014).

47. F.L. Mota, N. Bergeon, D. Tourret, A. Karma, R. Trivedi, and B. Billia, Acta Mater. 85, 362-377 (2015).

48. V. Fallah, M. Amoorezaei, N. Provatas, S.F. Corbin, and A. Khajepour, Acta Mater. 60, 1633-1646 (2012).

49. D. Montiel, L. Liu, L. Xiao, Y. Zhou, and N. Provatas, Acta Mater. 60, 5925-5932 (2012).

50. I. Steinbach, Acta Mater. 57, 2640-2645 (2009).

51. G. Zimmermann, L. Sturz, B. Billia, N. Mangelinck-Noël, D.R. Liu, H. Nguyen-Thi, N. Bergeon, Ch-A Gandin, D.J. Browne, C. Beckermann, D. Tourret, and A. Karma, Mater. Sci. Forum 790, 12-21 (2014). 